\title{
Distribution of human papilloma virus genotype prevalence in invasive cervical carcinomas and precancerous lesions in the Yangtze River Delta area, China
}

Hongyun WANG ${ }^{1,2}$, Xiaodong $\mathrm{CHENG}^{1}$, Jing $\mathrm{YE}^{1}$, Xiuyun $\mathrm{XU}^{2}$, Ying $\mathrm{HONG}^{2}$, Long SUI ${ }^{3}$, Zhixue YOU ${ }^{4}$ and Xing $\mathrm{XIE}^{1^{*}}$

\begin{abstract}
Background: This study aimed to provide more information for cancer prevention strategies by determining the distribution of human papilloma virus (HPV) genotype prevalence in invasive cervical carcinoma (ICC) and precancerous lesion patients in the Yangtze River Delta area in China.

Methods: This multi-centre descriptive cross-sectional study involves four university hospitals in the Jiangzhehu area. Women with histologically confirmed cervical intraepithelial neoplasia (CIN) 1, CIN2, CIN3 or ICC who were diagnosed and treated in the four selected hospitals between February 2012 and April 2014 were eligible for recruitment. The average age of the patients was $40.93 \pm 11.87$ years old, among whom the youngest was 17 years old and the oldest was 76 years old.Those with immunodeficiency diseases or a previous history of cancer or CIN were excluded. HPV genotyping was performed by a central laboratory. The distribution and age and disease specificity of the HPV genotype prevalence were analysed.

Results: Of the 2181 collected samples, 251 were ICC and 1930 were CIN. The mean age of cervical cancer and CIN patients was $40.93 \pm 11.8$ years (range, 17-76 years). The five most commonly identified HPV types in each lesion class were as follows: CIN1: 52, 58, 16, 33, and CP; CIN2: 16, 58, 52, 33, and 31; CIN3: 16, 58, 33, 52, and 31; and ICC: 16, 58, 18, 52, and 33. CIN1 had an earlier age of onset (30-40 years) than CIN2, CIN3, and cervical cancer. The age of onset of cervical cancer exhibited two peaks at 40-44 and 50-54 years of age. In all infected patients, the frequency of HPV infection with a single type was $62.9 \%$, and with multiple types, it was $38.1 \%$. There was no difference in the frequencies of multiple types amongst the different cervical lesions.

Conclusions: The most prevalent genotypes in the investigated area (52,58, 16 and 18) justify the necessity of anti-HPV vaccination in teenagers and young girls under 24 years old in the Yangtze River Delta area in China. Infection with multiple high-risk HPV types versus single infection does not increase the risk for $\geq$ CIN2 in ICC development.
\end{abstract}

Keywords: Cervical intraepithelial neoplasia (CIN), Human papilloma virus (HPV), Invasive cervical cancer (ICC)

\footnotetext{
* Correspondence: xiex@zju.edu.cn

'Department of Gynecological Oncology, Women's Hospital, School of

Medicine, Zhejiang University, Zhejiang, China

Full list of author information is available at the end of the article
}

(c) The Author(s). 2018 Open Access This article is distributed under the terms of the Creative Commons Attribution 4.0 International License (http://creativecommons.org/licenses/by/4.0/) which permits unrestricted use, distribution, and reproduction in any medium, provided you give appropriate credit to the original author(s) and the source, provide a link to the Creative Commons license, and indicate if changes were made. The Creative Commons Public Domain Dedication waiver (http://creativecommons.org/publicdomain/zero/1.0/) applies to the data made available in this article, unless otherwise stated. 


\section{Background}

Cervical cancer is the third most common cancer amongst women worldwide, with an estimated 500,000 new cases annually and a $50 \%$ mortality rate[1]. Human papillomavirus (HPV), the most common sexually transmitted virus [2], has been identified as a major risk factor for both ICC and cervical intraepithelial neoplasia (CIN). CIN1 has the risk of developing into $\geq$ CIN2, and $\geq$ CIN2 has the risk of developing into ICC; $\geq$ CIN2 is also known as precancerous lesions. A recently developed HPV vaccine is effective in the prevention of cervical neoplasia. One quadrivalent and one bivalent vaccine targeting genital HPV have been effectively applied for the prevention of CIN in over 100 countries $[3,4]$. Additionally, a 9-valent vaccine is currently available. However, the effect of prophylactic vaccines is type-specific. Both currently licensed vaccines prevent infection of the most prevalent HPV types (types 16, 18, 6 , and 11), but they do not cover all high-risk HPV (HRHPV) types associated with cervical cancer. Furthermore, the effective prevention of HPV types covered by a vaccine may affect their distribution. Therefore, determining the distribution of HPV types before beginning a vaccination programme will provide baseline information enabling the evaluation of the effectiveness of mass vaccination and the identification of a change in type distribution. Because it is impossible to investigate all HPV types, clarification of the most carcinogenic types of HPV will provide important knowledge for the development of a new generation of HPV vaccines.

Although infection with HPV is common while ICC is rare, persistent infection with high-risk or oncogenic types increases the risk of developing cancer. International prevalence studies estimate that approximately $70 \%$ of ICCs are attributed to either HPV-16 or -18 [5-8]. A meta-analysis of 85 studies worldwide revealed that the overall rate of HPV infection in ICC patients was similar in all regions (83-89\%). HPV-16 was the predominant type in squamous cell carcinoma (SCC; 46-63\%) followed by HPV-18 (10-14\%). The predominant type in adenocarcinoma (ACa) and adenosquamous carcinoma (AdCa) was HPV-18 (37-41\%) followed by HPV-16 (26-36\%) [6]. HPV-16/18 accounted for $52 \%$ of infections in high-grade cervical lesions [9]. However, the type distribution of HPV infection varies widely across the world $[2,9,10]$. Elucidation of the type distribution of HPV will aid the development of public health policy programmes and provide evidence for the future selection of vaccines targeting HPV types common to a specific region. Despite these obvious benefits, data concerning HPV distribution are rarely reported in most underdeveloped countries or regions.

The Yangtze River Delta region (Jiangzhehu area) includes Jiangsu Province, Zhejiang Province, and Shanghai; it is the most economically developed region in China. The total GDP in the Yangtze River Delta area was approximately $\$ 1$ trillion USD in 2010 [11], and the per capita GDP has exceeded \$4000 USD since 2005 [12]. This provides strong economic support for the widespread use of the HPV vaccination. Additionally, the population possesses a high awareness of health issues and has the highest demand for self-expense vaccination in China. Considering the shortage of medical care in China, the $\mathrm{HPV}$ vaccine has not been widely used. However, it can be used in the economically developed Yangtze River Delta Region because of self-funded vaccination. Because clinical trials of both HPV vaccines are nearing completion in China, elucidating the type-specific distribution of HR-HPV in the region prior to the implementation of mass vaccination programmes is prudent and will provide scientific evidence for the future development of a new generation of vaccines suitable for this region.

In this cross-sectional study, cervical specimens from ICC and CIN patients residing in the Delta region were collected. HPV genotypes were determined, and the type distribution was analysed amongst the population. This study aimed to understand the distribution of HPV types and to provide comprehensive scientific evidence to aid the development of control strategies and prevent cervical cancer in the region.

\section{Methods \\ Material}

Suitable patients who visited one of four universityaffiliated hospitals within the region were enrolled in this study. These hospitals included the Women's Hospital, Zhejiang University School of Medicine, the Affiliated Drum Tower Hospital of Nanjing University School of Medicine, the Obstetrics and Gynaecology Hospital of Shanghai Fudan University, and the First Affiliated Hospital of Nanjing Medical University. The cases came from 4 centres of the Yangtze River Delta area in China. These four central hospitals represent the top hospitals of Zhejiang, Jiangsu and Shanghai. According to the number of patients with local illnesses, more than half of the local women in this area undergo abnormal and opportunistic screening for cervical cancer in the clinics of these four hospitals. Approximately $15 \%$ of the local population participated in cervical cancer screening. All hospitals have sufficient patient resources to reflect the HPV classification of cervical cancer and precancerous lesions and suitable clinical and laboratory facilities to undertake this study.

The study population comprised patients diagnosed with CIN1, CIN2, CIN3, or ICC based on pathology who were treated at one of the four aforementioned hospitals between February 2012 and April 2014. To be included in the study, patients were required to meet the 
following inclusion criteria: untreated histopathological diagnosis of CIN1, CIN2, CIN3, or ICC; no previous ICC or CIN history; negative for immunodeficiency diseases, including HIV; informed consent; and permanent residency in the Yangtze River Delta region. The average age of the patients was $40.93 \pm 11.87$ years old, among whom the youngest was 17 years old and the oldest was 76 years old. There were no patients younger than 16 years old. There were 10 excluded patients whose postoperative diagnoseis didn't accord with preoperative diagnoses (3 CIN1 cases, 2 CIN2 cases, 3 CIN3 cases,and 2 ICC cases).

Pathological diagnosis of cervical lesions was used as the gold standard, and the diagnosis was conducted and reviewed in each hospital independently. Uniform diagnostic review was not conducted. Diagnosis of cervical cytology was classified by the 2001 revised Bethesda system. Pathology experts interpreted the results as follows: absence of intraepithelial lesion or malignancy (WLM); atypical squamous cells of undetermined significance (ASCUS or AGUS); atypical squamous cells, high-grade intraepithelial lesions cannot be excluded (ASC-H); lowgrade squamous intraepithelial lesion (LSIL); high-grade squamous intraepithelial lesion (HSIL), including those caused by HPV infection; and SCC, ACa, and AdCa. Histological grading was based on Broder's classification.

\section{Methods research protocols}

A cross-sectional study design was employed to determine the distribution of HPV genotypes amongst ICC and CIN patients in the Delta region. According to the histopathological results, patients were divided into 4 groups: the CIN1, CIN2, CIN3 and ICC groups. The patient information was collected and then tested for HPV typing.

\section{Specimen collection}

All participating hospitals followed the same patient registration protocol and obtained the following information: patient name, assigned patient number, completed HPV patient information collection form (CRF), and signed informed consent. The survey was approved by the Medical Ethics Committee of Affiliated Obstetrics and Gynecology Hospital of Zhejiang University Medical School(or called Women's Hospital School Of Medicine Zhejiang University). Cervical cytology was conducted for all patients following a gynaecological examination. The study was conducted over a period of 26 months. For consistency of results and to ensure proficiency, all registrations were completed by a physician and a trained investigator at each hospital.

All participating hospitals sequentially registered patients, and physicians recorded all information in the HPV-CRF. According to the histopathological results, patients were divided into 4 groups: the CIN1, CIN2, CIN3 and ICC groups. The cervical exfoliated cells collected before operation were genotyped for HPV, 10 cases were excluded because of the inconformity of the pre- and post-operative diagnoses. A Cytobrush was inserted into the endocervical canal until the brush bent against the ectocervix. The brush was then rotated clockwise five times. After specimen collection, the brush head was placed into a collection vial that was labelled with the collection date, patient name, and patient number before the HPV types was checked. The storage vial with the brush head was stored immediately at $-20^{\circ} \mathrm{C}$. All specimens were sent to a central laboratory within weeks of collection for determination of the HPV genotype.

\section{Determination of HPV genotype}

HPV genotyping was conducted as reported previously [13].

\section{Real time-PCR for HR-HPV DNA}

An HPV GenoArray test was performed for the HR-HPV samples. The HPV genotype was determined by DNA amplification, flow-through hybridization, and a gene chip using the 21 HPV GenoArray Diagnostic Kit (HybriMax, Chaozhou Hybribio Limited Corp., Chaozhou, China) according to the manufacturer's instructions. Detailed protocols for this assay have been described previously [13]. The gene chip contained type-specific oligonucleotides immobilized on a nylon membrane. The chip could identify 13 HR-HPVs $(16,18,31,33,35,39,45$, 51, 52, 56, 58, 59, and 68), five low-risk HPVs (LR-HPVs) $(6,11,42,43$, and 44), and other HPV types found in Chinese populations (53, 66, and 81/CP8304). The results were detected by colorimetric changes on the chip under direct visualization.

All results were verified using the 37 HPV GenoArray Diagnostic Kit from the same company. The chip could identify 15 HR-HPVs $(16,18,31,33,35,39,45,51,52$, $53,56,58,59,66$, and 68) and 24 LR-HPVs $(6,11,26$, $34,40,42,43,44,54,55,57,61,67,69,70,71,72,73$, 82, 83, 84, and CP8304).

\section{Statistical analysis}

A database was established using Excel 2003, and the results were analysed using SPSS 17.0 software (SPSS Inc., Chicago, IL, USA). A Chi-square test was used for the counting analysis, and a $t$-test was used for variable data. A $p$ value of $<0.05$ was considered significant.

\section{Results}

Distribution of HPV types in CIN and ICC women from the Yangtze River Delta region

A total of 2181 qualified specimens were collected. The specimens included 251 ICC cases and 1930 CIN cases. 
HPV infection was not detected in 200 cases. Infection with one, two, three, four, five, six, and eight types of HPV was detected in 1372 cases, 454 cases, 119 cases, 29 cases, 5 cases, 1 case, and 1 case, respectively. One 50-year-old patient with a CIN2 pathological diagnosis tested positive for HPV types 52, 39, 45, 51, 35, 53, 66, and CP8304.The distribution of HPV infection with a single type and multiple types in the study population from the Yangtze River Delta region is shown in Table 1 and Fig. 1.

Table 1 Distribution of HPV infection with a single type and multiple types in women from the Yangtze River Delta region

\begin{tabular}{|c|c|c|c|c|c|c|c|c|c|c|}
\hline \multirow[t]{2}{*}{ Types } & \multicolumn{2}{|c|}{ CIN1 } & \multicolumn{2}{|c|}{ CIN2 } & \multicolumn{2}{|c|}{ CIN3 } & \multicolumn{2}{|l|}{ ICC } & \multicolumn{2}{|l|}{ Total } \\
\hline & $n$ & (\%) & $n$ & (\%) & $n$ & $(\%)$ & $n$ & (\%) & $n$ & (\%) \\
\hline Total & 751 & & 425 & & 753 & & 252 & & 2181 & \\
\hline Total HPV (+) & 666 & (88.68) & 392 & $(92.24)$ & 701 & (93.09) & 222 & (88.10) & 1981 & (90.83) \\
\hline 16 & 117 & $(17.57)$ & 135 & (34.44) & 411 & $(58.63)$ & 146 & $65.77)$ & 809 & $(40.84)$ \\
\hline 58 & 167 & $(25.08)$ & 95 & $(24.23)$ & 129 & $(18.40)$ & 20 & $(9.01)$ & 411 & $(20.75)$ \\
\hline 52 & 184 & $(27.63)$ & 75 & $(19.13)$ & 93 & $(13.27)$ & 19 & $(8.56)$ & 371 & (18.73) \\
\hline 33 & 59 & $(8.86)$ & 47 & (11.99) & 104 & (14.84) & 15 & $(6.76)$ & 225 & (11.36) \\
\hline 18 & 41 & $(6.16)$ & 20 & $(5.10)$ & 32 & $(4.56)$ & 27 & $(12.16)$ & 120 & $(6.06)$ \\
\hline 31 & 38 & $(5.71)$ & 29 & $(7.40)$ & 54 & $(7.70)$ & 9 & $(4.05)$ & 130 & $(6.56)$ \\
\hline 53 & 38 & $(5.71)$ & 25 & $(6.38)$ & 37 & $(5.28)$ & 12 & $(5.41)$ & 112 & $(5.65)$ \\
\hline 68 & 34 & $(5.11)$ & 16 & $(4.08)$ & 27 & $(3.85)$ & 10 & $(4.50)$ & 87 & $(4.39)$ \\
\hline 39 & 32 & $(4.80)$ & 11 & $(2.81)$ & 12 & $(1.71)$ & 7 & (3.15) & 62 & (3.13) \\
\hline 51 & 31 & $(4.65)$ & 11 & $(2.81)$ & 6 & $(0.86)$ & 2 & $(0.90)$ & 50 & $(2.52)$ \\
\hline 66 & 30 & $(4.50)$ & 8 & $(2.04)$ & 10 & $(1.43)$ & 1 & $(0.45)$ & 49 & $(2.47)$ \\
\hline 56 & 30 & $(4.50)$ & 4 & $(1.02)$ & 7 & $(1.00)$ & 1 & $(0.45)$ & 42 & $(2.12)$ \\
\hline 35 & 10 & $(1.50)$ & 8 & $(2.04)$ & 5 & $(0.71)$ & 1 & $(0.45)$ & 24 & $(1.21)$ \\
\hline$C P$ & 49 & $(7.36)$ & 25 & $(6.38)$ & 28 & (3.99) & 10 & $(4.50)$ & 112 & $(5.65)$ \\
\hline 11 & 15 & $(2.25)$ & 5 & $(1.28)$ & 9 & $(1.28)$ & 4 & $(1.80)$ & 33 & $(1.67)$ \\
\hline 6 & 7 & $(1.05)$ & 9 & $(2.30)$ & 5 & $(0.71)$ & 1 & $(0.45)$ & 22 & $(1.11)$ \\
\hline 42 & 7 & $(1.05)$ & 2 & $(0.51)$ & 5 & $(0.71)$ & 3 & $(1.35)$ & 17 & $(0.86)$ \\
\hline
\end{tabular}

Note: Upright letters indicate HR-HPV' italics indicate LR-HPV. HPV types with the frequency less than $1 \%$ are not shown

The proportion of HPV16 type in ICC is significantly different from that of other high-risk type HPV, $P<0.01$ (HPV58, $X^{2}=173.40 ; H P V 52, X^{2}=176.60 ; H P V 33, X^{23}=189.83$; HPV18, $\left.X^{2}=152.27 ; H P V 31, X^{2}=211.23 ; H P V 53, X^{2}=200.26 ; H P V 68, X^{2}=207.55 ; H P V 39, X^{2}=218.76 ; H P V 51, X^{2}=238.59 ; H P V 66, X^{2}=242.74 ; H P V 56, X^{2}=242.74 ; H P V 35, X^{2}=242.74\right)$ The proportion of HPV18 type in ICC is significantly different from that of other high-risk type HPV, $p<0.01$ (HPV16, $X^{2}=152.27 ; H P V 31, X^{2}=11.13$; HPV53, $x^{2}=7.16$; HPV68, $X^{2}=9.68 ;$ HPV39, $\left.X^{2}=14.47 ; H P V 51, X^{2}=26.17 ; H P V 66, X^{2}=29.25 ; H P V 56, X^{2}=29.25 ; H P V 35, X^{2}=29.25\right)$, except HPV58,52 and 33 type (HPV58, $X^{2}=1.32, P>0.05$, HPV52, $x^{2}=1.76, p>0.05 ; \mathrm{HPV} 33, x^{2}=4.29,0.01<\mathrm{p}<0.05$ )

The proportion of HPV58 type in ICC is significantly different from that of other high-risk type HPV, $P<0.01$ (HPV16, $X^{2}=173.40 ; H P V 39, X^{2}=7.58 ; H P V 51, X^{2}=17.60$; HPV66, $X^{2}=20.49 ;$ HPV56, $x^{2}=20.49 ;$ HPV35, $x^{2}=20.49$ ), except HPV52,33,18,53 type (HPV52, $\left.X^{2}=0.03 ; H P V 33, x^{2}=0.88 ; H P V 18, X^{2}=1.32 ; H P V 53, x^{2}=2.44\right), p>0.05$ and HPV31, 68 type (HPV31, $x^{2}=5.08 ;$ HPV68, $\left.x^{2}=4.07\right), 0.01<\mathrm{p}<0.05$

The proportion of HPV16 type in CIN3 is significantly different from that of other high-risk type HPV, $P<0.01$ (HPV58, $X^{2}=257.32 ; H P V 52, X^{2}=336.43 ; H P V 33, X^{23}=310.65$; HPV18, $\left.X^{2}=509.30 ; H P V 31, X^{2}=440.58 ; H P V 53, X^{2}=492.83 ; H P V 68, X^{2}=525.97 ; H P V 39, X^{2}=579.00 ; H P V 51, X^{2}=601.28 ; H P V 66, X^{2}=586.26 ; H P V 56, X^{2}=597.56 ; H P V 35, X^{2}=605.29\right)$ The proportion of HPV58 type in CIN3 is significantly different from that of other high-risk type HPV, P<0.01 (HPV16, $x^{2}=257.32 ; \mathrm{HPV} 52, x^{2}=7.43 ; \mathrm{HPV} 18, x^{2}=70.97 ; \mathrm{HPV} 31, x^{2}=37.99$; HPV53, $X^{2}=62.09 ;$ HPV68, $X^{2}=80.61 ;$ HPV39, $X^{2}=115.96 ;$ HPV51, $X^{2}=133.10 ;$ HPV66, $X^{2}=121.39 ;$ HPV56, $\left.X^{2}=130.14 ; H P V 35, X^{2}=136.33\right)$, except HPV33 (HPV33, $\left.X^{2}=3.44\right), p>0.05$ The proportion of HPV52 type in CIN3 is significantly different from that of other high-risk type HPV, P>0.01 (HPV16, $X^{2}=336.43 ; \mathrm{HPV} 58, X^{2}=7.43 ; \mathrm{HPV} 18, X^{2}=35.17 ; \mathrm{HPV} 31$,

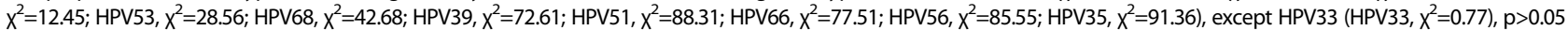
The proportion of HPV16 type in CIN2 is significantly different from that of other high-risk type HPV, $P<0.01$ (HPV58, $X^{2}=10.69 ; H P V 52, X^{2}=25.40 ; H P V 33, X^{2}=60.08 ; H P V 18$, $\left.X^{2}=115.33 ; H P V 31, X^{2}=93.92 ; H P V 53, X^{2}=103.00 ; H P V 68, X^{2}=125.96 ; H P V 39, X^{2}=140.27 ; H P V 51, X^{2}=140.27 ; H P V 66, X^{2}=149.58 ; H P V 56, X^{2}=162.71 ; H P V 35, X^{2}=149.58\right)$ The proportion of HPV58 type in CIN2 is significantly different from that of other high-risk type HPV, $P<0.01$ (HPV16, $X^{2}=10.69 ; H P V 33, X^{2}=21.47 ; H P V 18, X^{2}=62.14 ; H P V 31$, $\left.X^{2}=45.21 ; H P V 53, X^{2}=52.23 ; H P V 68, X^{2}=70.00 ; H P V 39, X^{2}=83.39 ; H P V 51, X^{2}=83.39 ; H P V 66, X^{2}=91.71 ; H P V 56, X^{2}=103.77 ; H P V 35, X^{2}=91.71\right)$, except HPV52 (HPV52, $\left.X^{2}=3.26\right), p>0.05$ The proportion of HPV52 type in CIN2 is significantly different from that of other high-risk type HPV, $P<0.01$ (HPV16, $X^{2}=25.40 ; H P V 33, X^{2}=8.25 ; H P V 18, X^{2}=39.29 ; H P V 31$, $\left.X^{2}=25.41 ; H P V 53, X^{2}=31.04 ; H P V 68, X^{2}=46.92 ; H P V 39, X^{2}=57.95 ; H P V 51, X^{2}=57.95 ; H P V 66, X^{2}=65.58 ; H P V 56, x^{2}=76.93 ; H P V 35, X^{2}=65.58\right)$, except HPV58 (HPV58, $\left.x^{2}=3.26\right), p>0.05$ The proportion of HPV16 type in CIN1 is significantly different from that of other high-risk type HPV, P $<0.01$ (HPV58, $X^{2}=12.62 ; H P V 52, X^{2}=21.72 ; H P V 33, X^{2}=24.84$; HPV18, $x^{2}=46.75 ;$ HPV31, $x^{2}=51.35 ;$ HPV53, $x^{2}=51.35 ;$ HPV68, $x^{2}=57.98 ;$ HPV39, $x^{2}=61.64 ;$ HPV51, $x^{2}=63.47 ;$ HPV66, $x^{2}=65.34 ;$ HPV56, $x^{2}=65.34 ;$ HPV35, $x^{2}=112.42$ ) The proportion of HPV58 type in CIN1 is significantly different from that of other high-risk type HPV, $P<0.01$ (HPV16, $X^{2}=12.62 ; H P V 33, X^{2}=70.11$; HPV18, $X^{2}=101.98$; HPV31, $X^{2}=108.17 ;$ HPV53, $X^{2}=108.17 ;$ HPV68, $X^{2}=116.84 ;$ HPV39, $X^{2}=121.53 ;$ HPV51, $X^{2}=123.84 ;$ HPV66, $X^{2}=126.19 ;$ HPV56, $X^{2}=126.19 ;$ HPV35, $\left.X^{2}=181.18\right)$, except HPV52 (HPV52, $\left.x^{2}=1.26\right), p>0.05$

The proportion of HPV52 type in CIN1 is significantly different from that of other high-risk type HPV, P<0.01 (HPV16, $X^{2}=21.72 ; H P V 33, X^{2}=88.69 ; H P V 18, X^{2}=123.28$; HPV31, $X^{2}=129.88 ; H P V 53, X^{2}=129.88 ; H P V 68, X^{2}=139.10 ; H P V 39, X^{2}=144.06 ; H P V 51, X^{2}=146.50 ; H P V 66, X^{2}=148.98 ;$ HPV56, $X^{2}=148.98 ;$ HPV35, $\left.X^{2}=206.04\right)$, except HPV58 (HPV58, $\left.X^{2}=1.26\right), p>0.05$ 


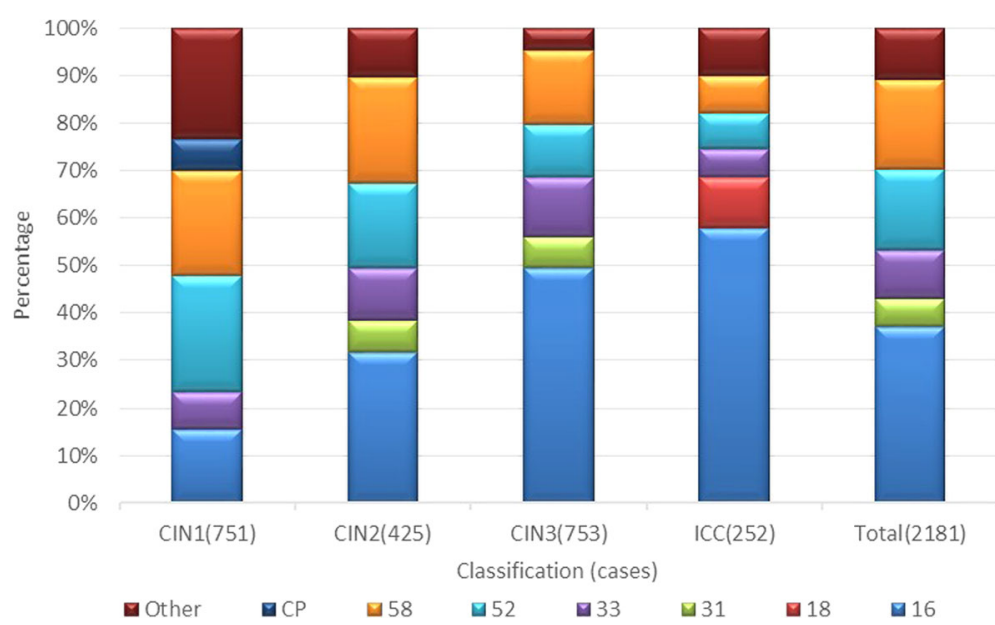

Fig. 1 Distribution of HPV infection with a single type and multiple types in women from the Yangtze River Delta region. (HPV)16, 18, 31, 33, 52, cp, 58, Other(\%)

The five most prevalent HPV types in ICC and its precursor patients from the Yangtze River Delta region were $16,58,52,33$, and 31 . Of these, the most prevalent types were 52, 58, 16, 33, and CP8304 in CIN 1; 16, 58, 52, 33, and 31 in CIN 2; 16, 58, 33, 52, and 31 in CIN 3; and $16,18,58,52$, and 33 in ICC.

The distribution of infection with a single HPV type in the study population from the Yangtze River Delta region is shown in Fig. 2.

The five most prevalent HPV types involving a single infection were $16,58,52,33$, and 31 in ICC and CIN patients from the Yangtze River Delta region. Of these, the five most prevalent HPV types of single infection were 52, 58, 16, 33, and CP8304 in CIN 1; 16, 58, 52, 33, and 31 in CIN 2; 16, 58, 33, 52, and 31 in CIN 3; and 16, $18,52,33$, and 31 in ICC.
The most prevalent HPV types were similar between single and multiple infections, suggesting that these types possess the strongest oncogenicity.

\section{Frequency of infection with a single or multiple HPV types in the Yangtze River Delta region}

In all infected patients, the frequency of HPV infection with a single type was $62.9 \%$, and with multiple types it was $38.1 \%$. The most common number of multiple infections involved two different types, and the maximum number of types was eight. There were no differences in the frequencies of multiple types amongst different cervical lesions, suggesting that increased numbers of HPV types do not elevate the risk for high-grade CIN in ICC development, as shown in Fig. 3.

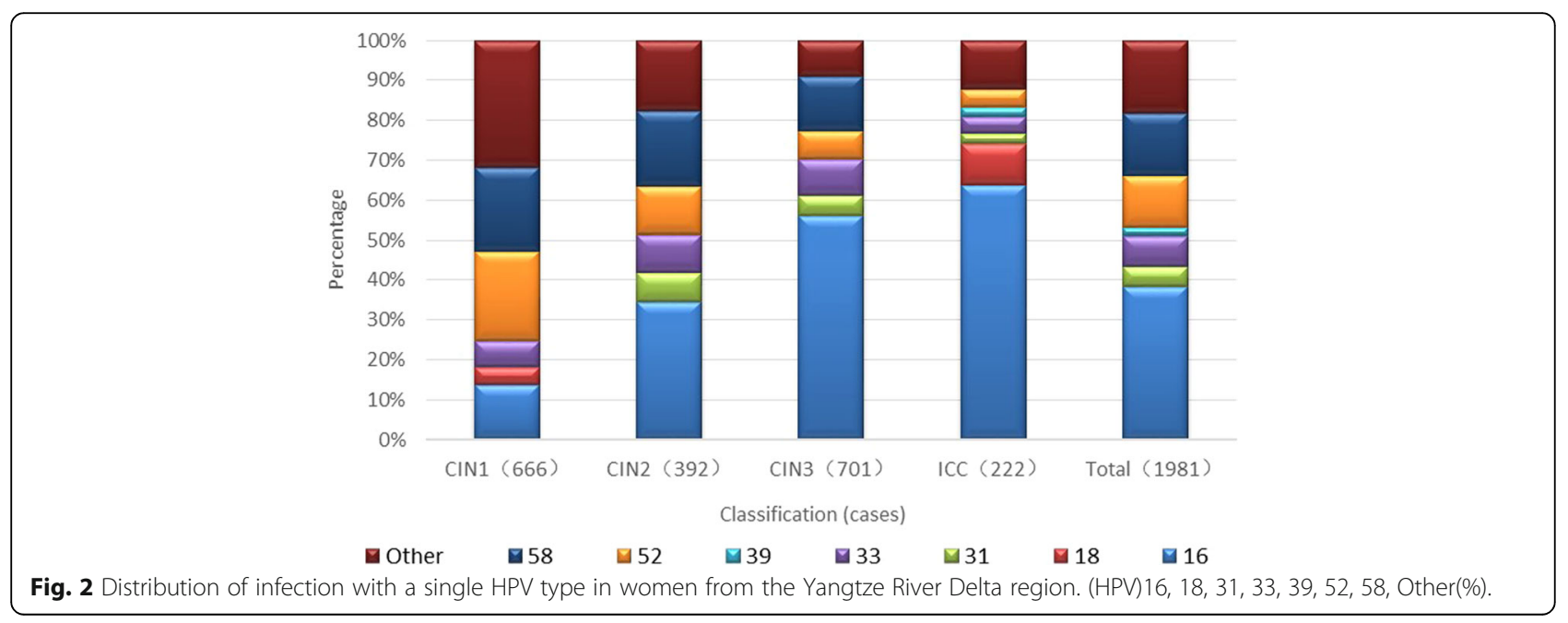




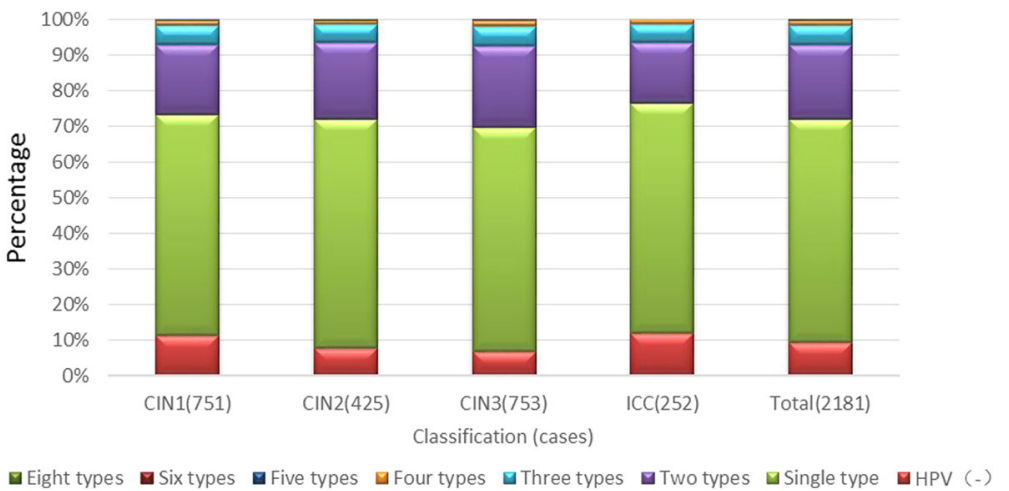

Fig. 3 Frequency of multiple HPV types stratified according to the pathological results in the three study regions. HPV(-), (HPV)Single type, Two types, Three types, Four types, Five types, Six types, Eight types (\%)

Frequency of multiple HPV types in the three regions (Table 2)

Regional distribution of disease and age in the Yangtze River Delta region (Table 3)

\section{Age distribution of CIN and ICC in Zhejiang Province} (Table 4)

\section{Age distribution of CIN and ICC patients in Jiangsu Province (Table 5)}

\section{Age distribution of CIN and ICC patients in Shanghai (Table 6)}

Age-stratified HPV distribution in the study population from the Yangtze River Delta region

HPV types with the frequency less than $1 \%$ are not shown.

Region-stratified HPV distribution in the study population from the Yangtze River Delta region

Age-stratified HPV distribution in the study population from the Yangtze River Delta region Fig 4. Age-stratified HPV distribution in CIN and ICC patients in the Delta Region (including multiple-type infection) HPV types with the frequency less than $1 \%$ are not shown.
HPV types with a frequency less than $1 \%$ are not shown.

HPV types with the frequency less than $1 \%$ are not shown.

There was no significant difference in HPV typing in Jiangsu, Zhejiang and Shanghai (Fig. 5). HPV types with the frequency less than $1 \%$ are not shown.

\section{Regional distribution of disease and age in the Yangtze River Delta region, Zhejiang Province, Jiangsu Province and shanghai}

In the Yangtze River Delta region, over 10\% of cervical cancer patients are under 35 years of age, and over $25 \%$ of patients with CIN and cervical cancer are under 35 years of age. The prevalence of CIN1 in the Delta region reached a peak in patients between 30 and 40 years of age, which is approximately 10 years earlier than the peak for other high-grade lesions. The prevalence of CIN3 and ICC peaked simultaneously and later than the peak for CIN2. ICC presented with two peaks at 40-44 and 50-54 years of age, as shown in Fig. 6. The Yangtze River Delta region $=$ YR,Zhejiang Province $=$ ZJ,Jiangsu Province $=$ JS,Shanghai $=$ SH,Of $=-$.For example (e.g.): Total of the Yangtze River Delta region(\%)= TotalYR,CIN1 of Zhejiang Province $=$ CIN1-ZJ.In Zhejiang Province, the incidence of cervical cancer in patients under 35 years old was $9.09 \%$. The incidence of ICC and

Table 2 Frequency of multiple HPV types in women from the three study regions

\begin{tabular}{|c|c|c|c|c|c|c|c|c|c|c|c|c|c|c|c|c|c|}
\hline \multirow{3}{*}{$\begin{array}{l}\text { Area } \\
\text { Zhejiang }\end{array}$} & \multirow{3}{*}{$\begin{array}{l}\text { TTotal } \\
941\end{array}$} & \multicolumn{2}{|c|}{ Not detected (HPV-) } & \multicolumn{14}{|c|}{ Types (\%) of HPV(+) } \\
\hline & & \multirow{2}{*}{$\begin{array}{l}\mathrm{n} \\
94\end{array}$} & \multirow{2}{*}{$\frac{(\%)}{(9.99)}$} & \multicolumn{2}{|c|}{ Single type } & \multicolumn{2}{|c|}{ Two types } & \multicolumn{2}{|c|}{ Three types } & \multicolumn{2}{|c|}{ Four types } & \multicolumn{2}{|c|}{ Five types } & \multicolumn{2}{|c|}{ Six types } & \multicolumn{2}{|c|}{ Eight types } \\
\hline & & & & 603 & $(64.08)$ & 183 & $(19.45)$ & 50 & $(5.31)$ & 8 & $(0.85)$ & 2 & $(0.21)$ & 1 & $(0.11)$ & 0 & $(0.00)$ \\
\hline Jiangsu & 784 & 69 & $(8.80)$ & 473 & $(60.33)$ & 170 & $(21.68)$ & 49 & $(6.25)$ & 19 & $(2.42)$ & 3 & $(0.38)$ & 0 & $(0.00)$ & 1 & $(0.13)$ \\
\hline Shanghai & 456 & 37 & $(8.11)$ & 296 & $(64.91)$ & 102 & $(22.37)$ & 19 & $(4.17)$ & 2 & $(0.44)$ & 0 & $(0.00)$ & 0 & $(0.00)$ & 0 & $(0.00)$ \\
\hline Total & 2181 & 200 & $(9.17)$ & 1372 & $(62.91)$ & 454 & $(20.82)$ & 119 & $(5.46)$ & 29 & $(1.33)$ & 5 & $(0.23)$ & 1 & $(0.05)$ & 1 & $(0.05)$ \\
\hline
\end{tabular}

The incidence of multiple-type HPV infections in Jiangsu Province was significantly higher $(p<0.05)$ than in either Zhejiang Province or Shanghai 
Table 3 Age distribution of patients with cervical lesions in the Yangtze River Delta region

\begin{tabular}{|c|c|c|c|c|c|c|c|c|c|c|}
\hline \multirow[t]{2}{*}{ Age (years) } & \multicolumn{2}{|l|}{ Total } & \multicolumn{2}{|c|}{ CIN1 } & \multicolumn{2}{|c|}{ CIN2 } & \multicolumn{2}{|l|}{ CIN3 } & \multicolumn{2}{|l|}{ ICC } \\
\hline & $\mathrm{n}$ & $(\%)$ & $\mathrm{n}$ & $(\%)$ & $\mathrm{n}$ & $(\%)$ & $\mathrm{n}$ & (\%) & $\mathrm{n}$ & (\%) \\
\hline$\leq 24$ & 43 & $(1.97)$ & 21 & $(2.80)$ & 12 & $(2.83)$ & 9 & $(1.19)$ & 1 & $(0.40)$ \\
\hline $25-29$ & 201 & $(9.22)$ & 95 & $(12.65)$ & 40 & $(9.43)$ & 60 & $(7.95)$ & 6 & $(2.39)$ \\
\hline $30-34$ & 338 & $(15.50)$ & 128 & (17.04) & 73 & $(17.22)$ & 116 & (15.36) & 21 & $(8.37)$ \\
\hline $35-39$ & 395 & $(18.11)$ & 134 & $(17.84)$ & 81 & (19.10) & 144 & (19.07) & 36 & (14.34) \\
\hline $40-44$ & 404 & $(18.52)$ & 134 & $(17.84)$ & 67 & $(15.80)$ & 150 & (19.87) & 53 & $(21.12)$ \\
\hline $45-49$ & 306 & $(14.03)$ & 99 & (13.18) & 60 & $(14.15)$ & 109 & (14.44) & 38 & (15.14) \\
\hline $50-54$ & 191 & $(8.76)$ & 45 & $(5.99)$ & 44 & (10.38) & 61 & (8.08) & 41 & (16.33) \\
\hline $55-59$ & 100 & $(4.59)$ & 29 & (3.86) & 21 & $(4.95)$ & 33 & $(4.37)$ & 17 & $(6.77)$ \\
\hline$\geq 60$ & 203 & $(9.31)$ & 66 & $(8.79))$ & 26 & $(6.13)$ & 73 & $(9.67)$ & 38 & (15.14) \\
\hline Total & 2181 & $(100)$ & 751 & (100) & 424 & (100) & 755 & (100) & 251 & $(100)$ \\
\hline
\end{tabular}

In the Yangtze River Delta region, over $10 \%$ of cervical cancer patients are under 35 years of age, and over $25 \%$ of patients with CIN and cervical cancer are under 35 years of age

CIN in patients under 35 years old was $26.05 \%$. The age distributions of patients with CIN1, CIN2, and ICC in Zhejiang Province all showed two peaks. The first peaks for CIN1 and CIN2 were 10 years earlier than that for ICC, and the first peak for CIN3 was 5 years earlier than that for ICC.In Jiangsu Province, the incidence of cervical cancer in patients less than 35 years old was 18 . $05 \%$. The incidence of ICC and CIN in patients less than 35 years old was $30.1 \%$. The age distribution of patients with ICC showed two peaks in Jiangsu Province. The first peak of Jiangsu Province for ICC was 5 years earlier than those of Zhejiang Province and Shanghai.In Shanghai, the incidence of cervical cancer in patients less than 35 years old was $4 \%$. The incidence of ICC and CIN in patients less than 35 years old was $21.93 \%$.The age distribution of patients with ICC showed two peaks in Shanghai. The prevalence of cervical lesions in patients less than 35 years old in Jiangsu Province was significantly higher $(x 2=9.75, \mathrm{p}<0.01)$ than that in Shanghai. The age distribution of patients with ICC also showed two peaks in Shanghai, whereas those of CIN1 and CIN2 were 5 years earlier than that of ICC.

The Yangtze River Delta region $=$ YR,Zhejiang Province $=\mathrm{Z}$, ,jiangsu Province $=\mathrm{JS}$, Shanghai $=\mathrm{SH}$, Of $=-$.For example (e.g.): Total of the Yangtze River Delta region $(\%)=$ Total-YR,CIN1 of Zhejiang Province = CIN1-ZJ.

In Zhejiang Province, the incidence of cervical cancer in patients under 35 years old was $9.09 \%$. The incidence of ICC and CIN in patients under 35 years old was $26.05 \%$.

The age distributions of patients with CIN1, CIN2, and ICC in Zhejiang Province all showed two peaks. The first peaks for CIN1 and CIN2 were 10 years earlier than that for ICC, and the first peak for CIN3 was 5 years earlier than that for ICC.

In Jiangsu Province, the incidence of cervical cancer in patients less than 35 years old was $18.05 \%$. The

Table 4 Age distribution of CIN and ICC in Zhejiang Province

\begin{tabular}{|c|c|c|c|c|c|c|c|c|c|c|}
\hline \multirow{2}{*}{$\begin{array}{l}\text { Age } \\
\text { (years) }\end{array}$} & \multicolumn{2}{|c|}{ Total } & \multicolumn{2}{|l|}{ CIN1 } & \multicolumn{2}{|c|}{ CIN2 } & \multicolumn{2}{|c|}{ CIN3 } & \multicolumn{2}{|l|}{ ICC } \\
\hline & $n$ & (\%) & $n$ & (\%) & $n$ & (\%) & $n$ & (\%) & $n$ & (\%) \\
\hline$\leq 24$ & 17 & $(1.81)$ & 14 & (3.29) & 2 & $(3.28)$ & 0 & $(0.00)$ & 1 & $(0.65)$ \\
\hline $25-29$ & 89 & $(9.46)$ & 61 & $(14.35)$ & 6 & $(9.84)$ & 21 & (6.98) & 1 & $(0.65)$ \\
\hline $30-34$ & 140 & $(14.88)$ & 80 & (18.82) & 6 & (9.84) & 42 & (13.95) & 12 & (7.79) \\
\hline $35-39$ & 135 & (14.35) & 63 & $(14.82)$ & 3 & $(4.92)$ & 50 & (16.61) & 19 & (12.34) \\
\hline $40-44$ & 166 & $(17.64)$ & 75 & $(17.65)$ & 6 & $(9.84)$ & 48 & $(15.95)$ & 37 & $(24.03)$ \\
\hline $45-49$ & 143 & $(15.20)$ & 58 & $(13.65)$ & 10 & (16.39) & 49 & (16.28) & 26 & (16.88) \\
\hline $50-54$ & 71 & (7.55) & 10 & $(2.35)$ & 9 & $(14.75)$ & 27 & (8.97) & 25 & (16.23) \\
\hline $55-59$ & 47 & (4.99) & 15 & (3.53) & 10 & (16.39) & 16 & $(5.32)$ & 6 & $(3.90)$ \\
\hline$\geq 60$ & 133 & (14.13) & 49 & (11.53) & 9 & $(14.75)$ & 48 & $(15.95)$ & 27 & (17.53) \\
\hline Total & 941 & (100) & 425 & (100) & 61 & (100) & 301 & (100) & 154 & (100) \\
\hline
\end{tabular}


Table 5 Age distribution of CIN and ICC patients in Jiangsu Province

\begin{tabular}{|c|c|c|c|c|c|c|c|c|c|c|}
\hline \multirow{2}{*}{$\begin{array}{l}\text { Age } \\
\text { (years) }\end{array}$} & \multicolumn{2}{|c|}{ Total } & \multicolumn{2}{|c|}{ CIN1 } & \multicolumn{2}{|c|}{ CIN2 } & \multicolumn{2}{|l|}{ CIN3 } & \multicolumn{2}{|l|}{ ICC } \\
\hline & $\bar{n}$ & (\%) & $\mathrm{n}$ & (\%) & $\bar{n}$ & (\%) & $\mathrm{n}$ & (\%) & $\bar{n}$ & (\%) \\
\hline$\leq 24$ & 19 & $(2.42)$ & 2 & $(1.23)$ & 9 & $(3.70)$ & 8 & $(2.61)$ & 0 & $(0.00)$ \\
\hline $25-29$ & 69 & $(8.80)$ & 15 & (9.26) & 20 & $(8.23)$ & 29 & $(9.45)$ & 5 & $(6.94)$ \\
\hline $30-34$ & 148 & (18.88) & 27 & (16.67) & 54 & $(22.22)$ & 59 & $(19.22)$ & 8 & (11.11) \\
\hline $35-39$ & 167 & $(21.30)$ & 36 & $(22.22)$ & 53 & $(21.81)$ & 66 & $(21.50)$ & 12 & (16.67) \\
\hline $40-44$ & 145 & (18.49) & 32 & (19.75) & 39 & (16.05) & 65 & $(21.17)$ & 9 & (12.50) \\
\hline $45-49$ & 91 & (11.61) & 15 & $(9.26)$ & 28 & $(11.52)$ & 38 & (12.38) & 10 & (13.89) \\
\hline $50-54$ & 66 & $(8.42)$ & 15 & $(9.26)$ & 19 & $(7.82)$ & 21 & $(6.84)$ & 11 & (15.28) \\
\hline $55-59$ & 36 & $(4.59)$ & 9 & (5.56) & 10 & $(4.12)$ & 8 & $(2.61)$ & 9 & (12.50) \\
\hline$\geq 60$ & 43 & $(5.48)$ & 11 & (6.79) & 11 & $(4.53)$ & 13 & $(4.23)$ & 8 & (11.11) \\
\hline Total & 784 & (100) & 162 & (100) & 243 & (100) & 307 & (100) & 72 & (100) \\
\hline
\end{tabular}

In Jiangsu Province, the incidence of cervical cancer in patients less than 35 years old was $18.05 \%$. The incidence of ICC and CIN in patients less than 35 years old was $30.1 \%$

incidence of ICC and CIN in patients less than 35 years old was $30.1 \%$.

The age distribution of patients with ICC showed two peaks in Jiangsu Province. The first peak of Jiangsu Province for ICC was 5 years earlier than those of Zhejiang Province and Shanghai.

In Shanghai, the incidence of cervical cancer in patients less than 35 years old was $4 \%$. The incidence of ICC and CIN in patients less than 35 years old was $21.93 \%$.

The age distribution of patients with ICC showed two peaks in Shanghai. The prevalence of cervical lesions in patients less than 35 years old in Jiangsu Province was significantly higher $\left(X^{2}=9.75, p<0.01\right)$ than that in Shanghai. The age distribution of patients with ICC also showed two peaks in Shanghai, whereas those of CIN1 and CIN2 were 5 years earlier than that of ICC.

\section{Discussion}

Distribution of HPV types in the Yangtze River Delta region in CIN and ICC women

An excess of $120 \mathrm{HPV}$ types have been identified to date, and over 40 types are associated with genital diseases. HPV types are classified as either HR-HPV or LR-HPV based on their carcinogenic potential. HR-HPV is associated with CIN2/3 and ICC and includes types $16,18,31$, $33,35,45,51,52,53,56,58,59,66$, and 68 . LR-HPV is associated with genital warts and LSIL and includes types $6,11,42,43$, and 44 [10].

Our present study identified that the most common HR-HPV types in CIN and ICC patients from the Delta region are HPV-16, 58, 52, 33, and 31. The distribution of infected HPV types shows substantial differences amongst healthy women, CIN and ICC patients. In a study in Zhejiang Province, Ye et al. reported an HPV

Table 6 Age distribution of CIN and ICC patients in Shanghai

\begin{tabular}{|c|c|c|c|c|c|c|c|c|c|c|}
\hline \multirow[t]{2}{*}{ Age (years) } & \multicolumn{2}{|c|}{ Total } & \multicolumn{2}{|c|}{ CIN1 } & \multicolumn{2}{|c|}{ CIN2 } & \multicolumn{2}{|c|}{ CIN3 } & \multicolumn{2}{|c|}{ ICC } \\
\hline & $n$ & (\%) & $n$ & $(\%)$ & $n$ & (\%) & $n$ & (\%) & $n$ & (\%) \\
\hline$\leq 24$ & 7 & $(1.54)$ & 5 & (3.05) & 1 & $(0.83)$ & 1 & $(0.68)$ & 0 & $(0.00)$ \\
\hline $25-29$ & 43 & $(9.43)$ & 19 & (11.59) & 14 & (11.67) & 10 & $(6.80)$ & 0 & $(0.00)$ \\
\hline $30-34$ & 50 & (10.96) & 21 & $(12.80)$ & 13 & (10.83) & 15 & $(10.20)$ & 1 & $(4.00)$ \\
\hline $35-39$ & 93 & (20.39) & 35 & (21.34) & 25 & (20.83) & 28 & (19.05) & 5 & $(20.00)$ \\
\hline $40-44$ & 93 & (20.39) & 27 & $(16.46)$ & 22 & (18.33) & 37 & $(25.17)$ & 7 & (28.00) \\
\hline $45-49$ & 72 & (15.79) & 26 & (15.85) & 22 & (18.33) & 22 & $(14.97)$ & 2 & $(8.00)$ \\
\hline $50-54$ & 54 & (11.84) & 20 & $(12.20)$ & 16 & (13.33) & 13 & $(8.84)$ & 5 & $(20.00)$ \\
\hline $55-59$ & 18 & (3.95) & 5 & (3.05) & 1 & $(0.83)$ & 9 & $(6.12)$ & 3 & (12.00) \\
\hline$\geq 60$ & 26 & $(5.70)$ & 6 & (3.66) & 6 & $(5.00)$ & 12 & (8.16) & 2 & $(8.00)$ \\
\hline Total & 456 & (100) & 164 & (100) & 120 & (100) & 147 & (100) & 25 & $(100)$ \\
\hline
\end{tabular}

In Shanghai, the incidence of cervical cancer in patients less than 35 years old was $4 \%$. The incidence of ICC and CIN in patients less than 35 years old was $21.93 \%$ 


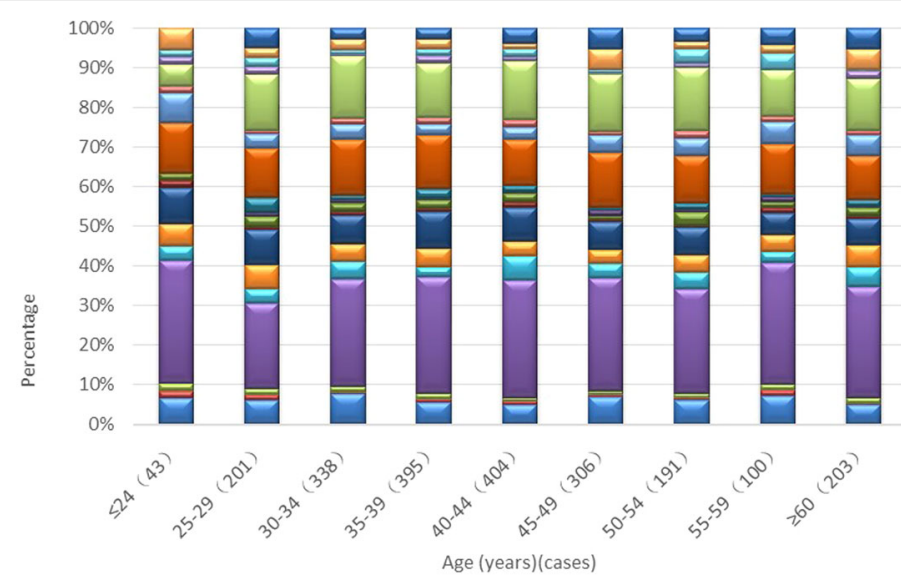

$\square C P \square 68 \square 66 \square 59 \square 58 \square 56 \square 53 \square 52 \square 51 \square 44 \square 39 \square 35 \square 33 \square 31 \square 18 \square 16 \square 11 \square 6 \square H P V(-)$

Fig. 4 Age-stratified HPV distribution in CIN and ICC patients in the Delta Region (including multiple-type infection). HPV(-), (HPV)6, 11, 16, 18, 31, $33,35,39,44,51,52,53,56,58,59,66,68, \mathrm{cp}(\%)$

prevalence of $13.3 \%$ using a cervical pap test. In the same population, the prevalence of HR-HPV was $10.2 \%$ [14]. The prevalence of individual HPV types was $3.1 \%$, $2.5 \%, 2.1 \%, 1 \%$, and $0.9 \%$ for types $52,16,58,68$, and 81 , respectively. HPV 16 and 18 accounted for $3.1 \%$ of all samples, whereas other types accounted for $3.5 \%(n=$ 174). In a study from Nanjing, Jiangsu Province, Hong et al. reported overall prevalence of HPV DNA amongst pregnant women of $13.4 \%$ (422/3139). The most frequently detected HPV genotypes were HPV-16 (29.6\%, $125 / 422),-18$ (14.7\%, 62/422), and - 58 (14.2\%, 60/422) [13]. The paper with the investigation of Ye and Hong in healthy women and cervical cancer HPV type is not exactly the same.

The distribution of infected HPV types shows substantial regional differences amongst CIN and ICC patients. Yang et al. previously investigated 1715 cervical neoplasia outpatients and identified HPV infection in $57.1 \%$ of those patients. They also reported that $76.9 \%$ of cancer patients were HPV-positive whereas $98.1 \%$ of CIN3 patients were HPV-positive. The most common types of HPV infection in these patients were HPV-16, 58, 52, 33 , and 31 [15]. Tang et al. found an HPV infection rate of $25.2 \%$ in patients with cervical disease, reflecting the trend of increased incidence in recent years. The most common HR-HPV types detected by Tang et al. were 16 , $58,33,52$, and 18 , with the distribution of HPV infection showing population differences and indicating that patients infected with a single HPV type may be more vulnerable to carcinogenesis [16]. In a randomized study, Munoz et al. found that the distribution of HPV types in the general population was similar to that in cervical cancer patients, with the most common being $16,18,45$, $31,58,33$, and 35 [10].

A meta-analysis of the HPV type distribution identified a total of 14,595 and 7094 cases of ICC and HSIL, respectively [9]. Worldwide, HPV16 was the most common type in ICC, followed by HPV18. In Asia, they were

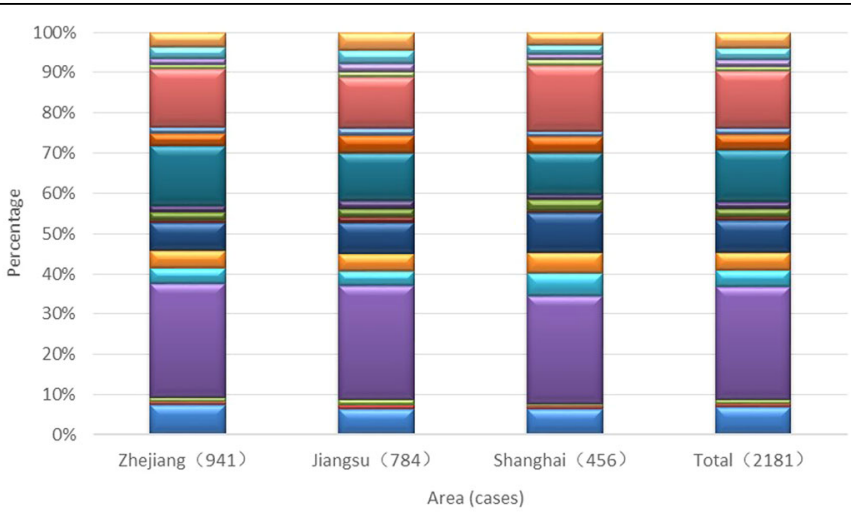

$\square \mathrm{CP} \square 68 \square 66 \square 59 \square 58 \square 56 \square 53 \square 52 \square 51 \square 39 \square 35 \square 33 \square 31 \square 18 \square 16 \square 11 \square 6 \square \mathrm{HPV}$

Fig. 5 Comparison of HPV distributions in the three study regions (including multiple-type infections). HPV(-), (HPV)6, 11, 16, 18, 31, 33, 35, 39, 44, $51,52,53,56,58,59,66,68, \mathrm{cp}(\%)$ 


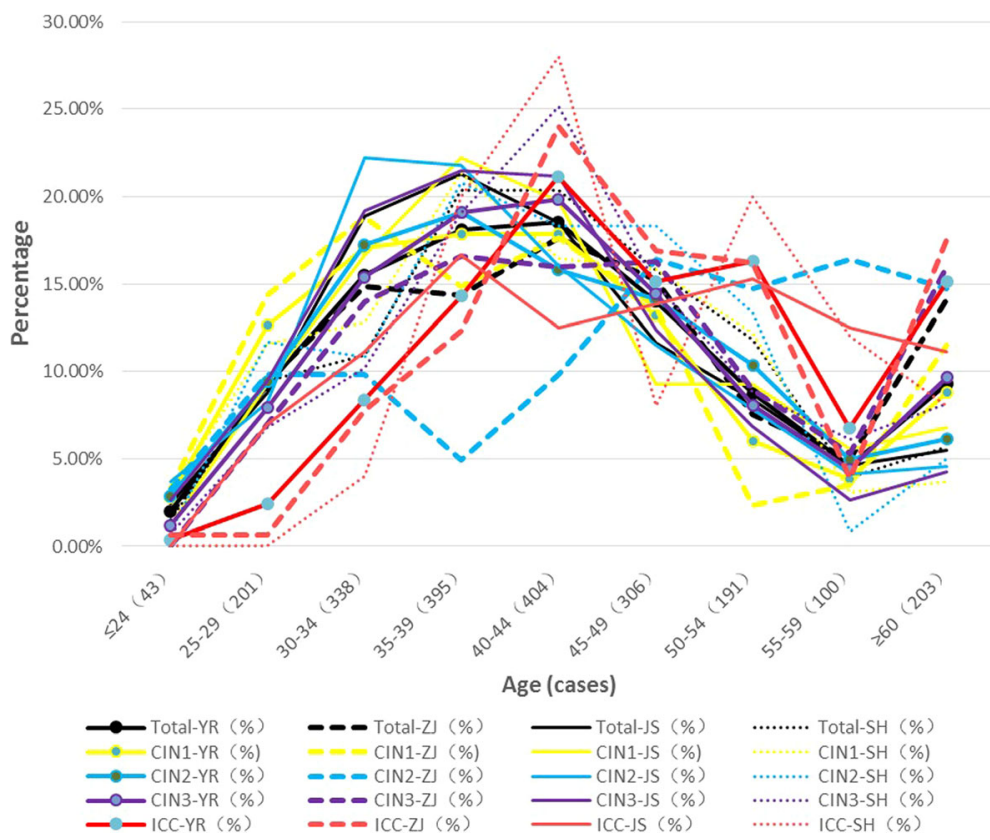

Fig. 6 Statistical age (years) distribution of cervical lesions in the Yangtze River Delta region,Zhejiang Province, Jiangsu Province and Shanghai. ICC, CIN3, CIN2, CIN1, Total (\%)

$16,18,58,33,52,45,31$, and 35 . In Asia, the HPV types in HSIL were $16,58,52,18,51,33,31$, and 56 . In ICC, HPV16 was the most common, with HPV18 the second most common type in all continents. The combined HPV16/18 prevalence amongst ICC cases was $65-70 \%$. The next most common HPV types were the same in each continent, namely, HPV31, 33, 35, 45, 52, and 58; however, their relative importance differed somewhat by region. HPV18 was significantly more prevalent in adeno/adenosquamous carcinoma than in squamous cell carcinoma, with the reverse being true for HPV16, 31, 33, 52 and 58. Amongst HSIL cases, the HPV16/18 prevalence was $52 \%$. However, HPV16, 18, and 45 were significantly under-represented, and other high-risk HPV types were significantly over-represented in HSIL compared with those in ICC, suggesting differences in type-specific risks for progression.

\section{HPV vaccination has good prospects in Yangtze River Delta}

In the Yangtze River Delta region, the HPV type distribution of women aged over 35 years was similar to that of the HSIL type in women with CIN2 and CIN3 [17]. Furthermore, the distribution of the 5 sites was similar to that of HPV type. Moreover, the HPV type distribution in women aged 25-29 years was similar to the ratio of the first 5 columns of the HPV type distribution in LSIL (CIN1) women.

We hope that this cohort study provides information to support vaccination before the peak age of HPV infection. The best time to vaccinate women against HPV is before engaging in sexual activities, or at minimum prior to 13-24 years of age.

The HPV distribution frequency is correlated to some extent with pathogenicity. For example, women with persistent infection were more likely to have a higher pathogenicity. Our current findings suggested that in groups aged under 30 and over 40 years old, the highest frequency distribution was consistent with high pathogenicity and was observed in HPV types 16, 18, 58, 52, 33, and 31.

According to the most prevalent genotypes in our area, vaccination is a strong tool in the prevention of cervical cancer (especially for 16, 18 52, and 58, genotypes, from 9-valent HPV vaccine), and new vaccines are necessary to cover some genotypes that are missing from current formula.

In CIN patients, the most prevalent HPV type was HPV-52, and infections with multiple types were identified in 10\% of individuals in the Yangtze River Delta Region. Jiangsu Province was found to have the most multiple-type infections. Our findings differ from those of the Beijing University First Hospital, Sichuan University, and Munoz, which all suggest a regional difference in HPV distribution between CIN and ICC. Our study indicates that HPV types 58 and 52, in addition to types 16 and 18, play important roles in the development of cervical cancer in the Yangtze River Delta Region.

There was no significant difference in the number of sexual partners or sexual behaviour of women in 
different areas of the Yangtze River Delta region. With respect to the economy, the GDP of Jiangsu Province was greater than that of Shanghai, which was greater than that of Zhejiang Province, whereas the national per capita income was the highest in Shanghai, followed by Zhejiang Province and Jiangsu Province. The per capita income is relatively low in Jiangsu Province; to increase this, the region has observed a large influx of personnel, resulting in an increased likelihood of HPV infection. Moreover, investigations into cervical cancer screening suggest an overall lack of health care knowledge and indicate that women do not pay attention to screening and early diagnosis and treatment of disease. This appears to be most serious in Jiangsu Province, followed by Zhejiang Province and Shanghai Province. Comparing the differences in the most common HPV types together with aspects of infection in the three different regions, we can conclude that the Jiangsu area has a higher proportion of composite infection. This likely reflects the rapid economic development of Jiangsu Province, the relatively low per capita income, the increased flow of personnel, the lack of health care knowledge, and the low importance placed on cervical cancer screening and early diagnosis and treatment of disease.

\section{Conclusions}

HPV58 and HPV52, in addition to HPV16 and HPV18, may be involved in the occurrence of cervical cancer in the Yangtze River Delta area in China. Infection with multiple high-risk HPV types does not increase the risk for high-grade CIN in ICC development.

In the Yangtze River Delta Region, the peak age of the onset of cervical cancer was between 40 and 54 years, whereas that of CIN was between 30 and 39 years of age. On average, the peak age of onset of CIN was 510 years earlier than that of cervical cancer. The most common HPV types identified were 52, 58, 16, 33, and CP8304 in CIN1 patients; 16, 58, 52, 33, and 31 in CIN2 patients; $16,58,33,52$, and 31 in CIN3 patients; and 16, $18,58,52$, and 33 in ICC patients. The age at which infection with HR-HPV most commonly occurs is the same as that in CIN and is earlier than the peak onset of cervical cancer. This is consistent with current epidemiological models of viral carcinogenesis.

According with the most prevalent genotypes in our area, vaccination is a strong tool in the prevention of cervical cancer, and new vaccines are necessary to cover some genotypes that are missing from the current formula.

\section{Abbreviations}

CIN: Cervical intraepithelial neoplasia; HCll: Hybrid Capturell; HPV: Human papillomavirus; HR-HPV: High risk human papilloma virus; HSIL: High-grade squamous intraepithelial lesion; ICC: Invasive cervical cancer; JS: Jiangsu Province; LSIL: Low-grade squamous intraepithelial lesion; SH: Shanghai; YR: The Yangtze River Delta region; ZJ: Zhejiang Province

\section{Acknowledgements}

We wish to thank all of our colleagues at the Women's Hospital of Department of Gynaecological Oncology of the School of Medicine of Zhejiang University, the Nanjing Drum Tower Hospital of Obstetrics and Gynaecology of the Affiliated Hospital of Nanjing University Medical School, the Obstetrics and Gynaecology Hospital of Fudan University, and the First Affiliated Hospital of Nanjing Medical University of Obstetrics and Gynaecology, particularly Weiguo Lv, Bei Cheng, Yifan Cheng, Yunfeng Fu, Yeli Yao, and Ying Li.

Thanks to the HPV testing laboratory of HybriMax.

Thanks to the Edanz group and American Journal Experts group for editing and proofreading work.

\section{Funding}

Funding for the study was Professor Xie's subject: Prevaccination distribution of cervical human papillomavirus (HPV) types and their associations with invasive cervical cancer and its precursors in Yangtze River Delta Area of China. The Fund provided financial and staff support. The study's funding is also a source of Professor Hong's subject: Science and Technology Department of Jiangsu province science and technology support programme - social development issues (CHINA) (BE2012606). The Fund provided financial and staff support. All authors acknowledge the support. Those funding the authors had no role in writing any part of this manuscript.

The research and publication fees were covered by the projects of Professor Hong and Professor Xie.

Availability of data and materials

All data from this project survey data.

\section{Authors' contributions}

XXIE: corresponding author, conceived and designed the project, analysed data, and provided materials and reviewed the final paper. He is responsible for this project. HW: First author, participated in the recent part of the collection of data, analysed data, provided materials and wrote the final paper. XC, JY, XXU, $Y H, L S$, and $Z Y$ : participated in the recent part of the collection of data. All authors read and approved the final manuscript.

\section{Ethics approval and consent to participate}

This study was based on the statement of ethical principles in the Declaration of Helsinki. Accordingly, this study was reviewed and approved by the clinical research ethics committee of the Women's Hospital, Zhejiang University School of Medicine (Medical Ethics Committee of Affiliated Obstetrics and Gynecology Hospital of Zhejiang University Medical School)(19 Oct 2011). The study protocol was approved by the ethical review boards of the participating institutions, and signed consent was obtained from each patient.

\section{Competing interests}

The authors declare that they have no competing interests.

\section{Publisher's Note}

Springer Nature remains neutral with regard to jurisdictional claims in published maps and institutional affiliations.

\section{Author details}

${ }^{1}$ Department of Gynecological Oncology, Women's Hospital, School of Medicine, Zhejiang University, Zhejiang, China. ${ }^{2}$ Nanjing Drum Tower Hospital, the Affiliated Hospital of Nanjing University Medical School, Nanjing, China. ${ }^{3}$ Obstetrics and Gynecology Hospital of Fudan University, Fudan, China. ${ }^{4}$ The First Affiliated Hospital of Nanjing Medical University, Nanjing, China.

Received: 11 April 2017 Accepted: 3 April 2018

Published online: 27 April 2018

References

1. s2010) World Health Organization available from: URL:http://www.who.int/en/. 
2. Baseman JG, Koutsky LA. The epidemiology of human papillomavirus infections. J Clin Virol. 2005;32(Suppl 1):S16-24. Review. PubMed PMID: 15753008.

3. FUTURE II Study Group. Quadrivalent vaccine against human papillomavirus to prevent high-grade cervical lesions. N Engl J Med. 2007;356(19):1915-27. PubMed PMID: 17494925.

4. Paavonen J, Naud P, Salmerón J, Wheeler CM, Chow SN, Apter D, Kitchener H, Castellsague X, Teixeira JC, Skinner SR, Hedrick J, Jaisamrarn U, Limson G, Garland S, Szarewski A, Romanowski B, Aoki FY, Schwarz TF, Poppe WA, Bosch FX, Jenkins D, Hardt K, Zahaf T, Descamps D, Struyf F, Lehtinen M, Dubin G. HPV PATRICIA study group. Efficacy of human papillomavirus (HPV)-16/18 AS04-adjuvanted vaccine against cervical infection and precancer caused by oncogenic HPV types (PATRICIA): final analysis of a double-blind, randomized study in young women. Lancet. 2009;374(9686): 301-14. doi:10.1016/S0140-6736(09)61248-4. Epub 2009 Jul 6. Erratum in: Lancet. 2010 Sep 25;376(9746):1054. PubMed PMID: 19586656.

5. Khan MJ, Castle PE, Lorincz AT, Wacholder S, Sherman M, Scott DR, Rush BB, Glass AG, Schiffman M. The elevated 10-year risk of cervical precancer and cancer in women with human papillomavirus (HPV) type 16 or 18 and the possible utility of type-specific HPV testing in clinical practice. J Natl Cancer Inst. 2005;97(14):1072-9. PubMed PMID: 16030305.

6. Bulkmans NW, Berkhof J, Bulk S, Bleeker MC, van Kemenade FJ, Rozendaal L, Snijders PJ, Meijer CJ, POBASCAM Study Group. High-risk HPV type-specific clearance rates in cervical screening. Br J Cancer. 2007;96(9):1419-24. Epub 2007 Mar 6. PubMed PMID: 17342094; PubMed Central PMCID: PMC2360183

7. Naucler P, Ryd W, Törnberg S, Strand A, Wadell G, Hansson BG, Rylander E, Dillner J. HPV type-specific risks of high-grade CIN during 4 years of followup:a population-based prospective study. Br J Cancer. 2007;97(1):129-32. Epub 2007 Jun 5. PubMed PMID: 17551490; PubMed Central PMCID: PMC2359659.

8. Zhang L, Bi Q, Deng H, Xu J, Chen J, Zhang M, Mu X. Human papillomavirus infections among women with cervical lesions and cervical cancer in eastern China: genotype-specific prevalence and attribution. BMC Infect Dis. 2017;17(1):107. PubMed PMID: 28143439; PubMed Central PMCID: PMC5282745.

9. Smith JS, Lindsay L, Hoots B, Keys J, Franceschi S, Winer R, Clifford GM Human papillomavirus type distribution in invasive cervical cancer and high-grade cervical lesions: a meta-analysis update. Int J Cancer. 2007;121(3): 621-32. PubMed PMID: 17405118.

10. Muñoz N, Bosch FX, de Sanjosé S, Herrero R, Castellsagué X, Shah KV, Snijders PJ. Meijer CJ; International Agency for Research on Cancer multicenter cervical Cancer study group. Epidemiologic classification of human papillomavirus types associated with cervical cancer. N Engl J Med. 2003;348(6):518-27. PubMed PMID: 12571259.

11. Vision/Overall Grasp. Tianjin Economy.2010,2:78. http://www.cnki.net/=

12. xinhuanet.com. The Yangtze River Delta in 2004 the per capita GDP of more than $\$ 4000$. Xinhuanet, April 4, 2005. http://www.china.com.cn/chinese/ECc/829122.htm.

13. Hong Y, Li SQ, Hu YL, Wang ZQ. Survey of human papillomavirus types and their vertical transmission in pregnant women. BMC Infect Dis. 2013;13:109. https://doi.org/10.1186/1471-2334-13-109. PubMed PMID: 23446269; PubMed Central PMCID: PMC3598550.

14. Ye J, Cheng X, Chen X, Ye F, Lü W, Xie X. Prevalence and risk profile of cervical human papillomavirus infection in Zhejiang Province, Southeast China: a population-based study. Virol J. 2010;7:66. https://doi.org/10.1186/ 1743-422X-7-66. PubMed PMID: 20307327; PubMed Central PMCID: PMC2857835

15. Yingjie YANG, Jian ZHAO, Xueqian $\mathrm{LI}$, et al. The association between human papillomavirus (HPV)types infection and the cervical lesions.Chinese journal of. Clin Obstet Gynecol. 2006;7(4):253-6. http://www.cnki.net/KCMS/detail/ detail.aspx?QueryID=16\&CurRec=1\&recid=\&filename=FKLC200604004\&db dname $=$ CJFD2006\&dbcode $=C J F Q \& p r=\& u r l i d=\& y x=\& v=M D I$ MDBIWDdFM dXhZUzdEaDFUM3FUcldNMUZyQ1dVSTCtIWitadEZDam1WcjdKSXliSG」 iRzRIdGZNcTQ5RIIJUjg.

16. Xiao TANG, Fan YANG, Ying HE, et al. Analysis of prevalence of human papillomavirus infection and its correlation with cervical lesions. J Practical Obstetrics Gynecol. 2013;29(4):302-5. http://www.cnki.net/KCMS/detail/de tail.aspx?QueryID=32\&CurRec=1\&recid=\&filename=SFCZ201304023\&dbname $=C J F D 2013 \& d b c o d e=C J F Q \& p r=\& u r l i d=\& y x=\& v=M j E 3 M j Z H N E g 5 T E 1 x N D \|$ dWjRSOGVYMUx1eFITNORoMVQzcVRyV00xRnJdDWJMK2VaK1 p0RkNqblU3 NOXOaXZJZEw.
17. Hongyun WANG. Study on the distribution of HPV genotype prevalence in invasive cervical carcinoma and precancerous lesions in Yangtze River Delta area: China[D] Zhejiang University; 2014. http://kns.cnki.net/KCMS/detail/ detail.aspx?dbcode=CDFD\&dbname $=$ CDFDLAST2015\&filename $=1015539246$. nh\&v=MzA0MjFGMjZHN2E3RjIQdSXFaRWJQSVI4ZVgxTHV4WVM3RGgxVdDNx VHJXTTFGckNVUkxLZIp1UnJGeWpuVmJ6TFY.

\section{Ready to submit your research? Choose BMC and benefit from:}

- fast, convenient online submission

- thorough peer review by experienced researchers in your field

- rapid publication on acceptance

- support for research data, including large and complex data types

- gold Open Access which fosters wider collaboration and increased citations

- maximum visibility for your research: over $100 \mathrm{M}$ website views per year

At BMC, research is always in progress.

Learn more biomedcentral.com/submissions 